\title{
Assessing dietary intake during the transition to adulthood: a comparison of age-appropriate FFQ for youth/adolescents and adults
}

\author{
Nicole Larson*, Lisa Harnack and Dianne Neumark-Sztainer \\ Division of Epidemiology and Community Health, School of Public Health, University of Minnesota, \\ 1300 South Second Street, Suite 300, Minneapolis, MN 55454, USA
}

Submitted 1 April 2011: Accepted 31 July 2011: First published online 20 September 2011

\begin{abstract}
Objective: Assessing changes in dietary intake during the transition from adolescence to adulthood is challenging given the need for age-appropriate tools at different developmental stages. The present study investigated the comparability of intake estimates as assessed with the youth/adolescent and adult forms of Willett's FFQ.

Design: Young adults were first asked to complete the adult FFQ as part of a larger study, Project EAT-III (Eating and Activity in Teens and Young Adults). A stratified random sample of respondents was invited to complete the youth/adolescent FFQ by mail within a 3-week period.

Setting: Participants were members of a longitudinal cohort who completed baseline surveys (including the adolescent FFQ) at schools in Minneapolis/ St. Paul, Minnesota and completed Project EAT-III surveys online or by mail in 2008-2009.

Subjects: There were ninety-one men and 103 women (median age $=24 \cdot 6$ years) who completed both forms of the FFQ.

Results: The adolescent and adult forms did not provide comparable absolute intake estimates. However, with few exceptions, correlation coefficients between intake estimates were moderate $(r=0 \cdot 4-0 \cdot 6)$. Furthermore, the percentage of individuals classified into the same quartile rank category based on their responses to the adolescent and adult forms was $\geq 50 \%$ for fibre, vitamins A and E, and servings of fruit (excluding juice), vegetables, dairy, whole grains and soft drinks.

Conclusions: Although responses on the adolescent and adult FFQ cannot be compared to describe changes in absolute intake over time, these tools provide comparable intake rankings and may be used together in longitudinal studies to investigate influences on diet.
\end{abstract}

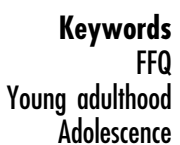

The transition from adolescence to young adulthood has been identified as a high-risk period for declines in diet quality and the development of obesity ${ }^{(1-3)}$. While the identification of effective prevention strategies will require an understanding of how dietary intake changes during the transition to young adulthood and what factors are influential, relatively little research has been conducted to investigate these questions ${ }^{(3)}$. There is a critical need for additional longitudinal studies among representative populations. However, the assessment of dietary intake in large samples of free-living individuals is difficult and quantifying change over time poses particular challenges as assessment tools designed for adults may not reflect typical dietary patterns during adolescence ${ }^{(4)}$.

Although the FFQ approach to assessing dietary intake is subject to measurement error, it offers a number of advantages for epidemiological studies of eating patterns among youth ${ }^{(4)}$. Given the high energy needs of adolescents in combination with typically unstructured eating patterns and the frequency of eating away from home throughout adolescence and young adulthood, requiring individuals to record their food and beverage consumption for multiple days may be onerous and limit participation $^{(5,6)}$. In addition, FFQ require less staff time and expense than other methods of dietary assessment and may be the only practical option for studies involving large samples ${ }^{(7)}$. To facilitate the use of the FFQ approach in adolescent populations, previous studies have reported on the reliability and validity of a Youth and Adolescent Questionnaire (YAQ) that was specifically designed for assessing dietary intake in youth aged 9 to 18 years ${ }^{(8,9)}$.

Longitudinal studies of dietary intake during late childhood and adolescence can use the YAQ to examine trends over time; however, this FFQ is likely not appropriate 
for assessing diet in adulthood. While the YAQ was designed according to the same format as the validated Willett FFQ for adults, no prior studies have examined the comparability of intake estimates determined by the YAQ and adult FFQ form ${ }^{(9)}$. The current study was therefore designed to examine whether the YAQ and adult FFQ form may be used together for the purpose of investigating changes in dietary intake during the transition from adolescence to young adulthood. Specifically, the study was designed to address two questions:

1. Do the YAQ and adult FFQ provide comparable estimates of absolute dietary intake?

2. Do the YAQ and adult FFQ form consistently rank individuals within a group according to their dietary intake?

The first question regarding absolute estimates has implications for examining changes in dietary intake patterns and assessing how intakes compare with dietary recommendations over time, and the second question regarding rank assignment is particularly relevant for research designed to identify factors that may influence intake patterns.

\section{Methods}

\section{Sample and study design}

Data for the present analysis were drawn from Project EAT-III (Eating and Activity in Teens and Young Adults), the third wave of a population-based study designed to examine dietary intake, physical activity and weightrelated issues in young adults. At baseline (Project EAT-I, 1998-1999), junior and senior high-school students at thirtyone public schools in the Minneapolis/St. Paul metropolitan area of Minnesota completed the Project EAT survey, anthropometric measurements and the $\mathrm{YAQ}^{(10,11)}$. Ten years later (2008-2009), original participants were mailed letters inviting them to complete online or paper versions of the Project EAT-III survey and the age-appropriate, adult form of the Willett $\mathrm{FFQ}^{(12)}$. Following survey completion, participants were mailed a \$US 50 gift card.

A subsample of young adult participants who completed the Project EAT-III survey and the adult FFQ were mailed invitations along with their gift card to complete a paper copy of the YAQ for an additional \$US $50 \mathrm{gift}$ card. To ensure a representative sample, participants in the main study were stratified by gender, age (20-25 or 26-31 years), race (white or non-white) and weight status (not overweight or overweight/obese) prior to random selection for recruitment. All study protocols were approved by the University of Minnesota's Institutional Review Board Human Subjects Committee. Participants indicated consent by returning completed questionnaires.

Completed YAQ were returned by 211 young adults (median age $=24 \cdot 6$ years, interquartile range $=22 \cdot 8-26 \cdot 0$ years) within 3 weeks, representing $68 \%$ of selected participants. A limited 3-week period was given for participants to respond in order to better capture differences attributable to measurement $v$. actual changes in dietary intake. Participants ( $n$ 17) who reported a biologically implausible level of total energy intake $(<2093 \mathrm{~kJ} / \mathrm{d}$ or $>20934 \mathrm{~kJ} / \mathrm{d}$ ) were excluded. The final sample for analysis included ninety-one men and 103 women who were diverse in terms of age (51\% aged 20-25 years), race ( $49 \%$ non-white) and weight status (56\% overweight or obese).

\section{Questionnaires and dietary analysis}

\section{Adult FFQ}

The 2007 grid form of the Willett semi-quantitative FFQ was used to assess usual dietary intake during the past year at Project EAT-III. This FFQ includes multi-vitamins, twenty-seven other dietary supplements (e.g. Ca, vitamin C, folic acid) and 151 foods with specified portion sizes ${ }^{(13)}$. For each food item, the questionnaire includes nine frequency categories that range from 'never or less than once per month' to 'six plus per day'. The reproducibility and validity of this FFQ were previously evaluated in adult men by comparing intake determined from the average of two 1-week diet records administered at an interval of approximately 6 months and intake determined from two FFQ administered at a 1 -year interval ${ }^{(14,15)}$. Oneyear reproducibility coefficients for unadjusted nutrient intake ranged from $0 \cdot 47$ to $0 \cdot 80^{(14)}$. The average correlation coefficient between energy-adjusted nutrient intake determined from the diet records and the FFQ administered in the same year was $0 \cdot 59^{(14)}$.

\section{Youth/adolescent FFQ}

The YAQ was used to assess usual dietary intake during the past year at Project EAT-I in the full sample and also at Project EAT-III in the subsample. While the YAQ is based on the Willett FFQ for adults, the developers made changes to the food list to better capture the diets of children and adolescents and modified the format to make the questionnaire simpler to complete ${ }^{(8,9)}$. Snack foods (e.g. fruit rollups, pudding) were emphasized on the YAQ along with other foods that are commonly consumed by young people (e.g. chicken nuggets, instant breakfast drink). In contrast to the horizontal format of the adult FFQ, questions and answers on the YAQ are presented in a vertical format such that each food item has an individual question and response. The YAQ assesses multi-vitamin use and 127 foods $^{(13)}$. Portion sizes were specified in natural units (e.g. one bunch of grapes, $1 \mathrm{yam} / \mathrm{sweet}$ potato) on the YAQ in contrast to the more specific portion sizes specified on the adult FFQ (e.g. $\frac{1}{2}$ cup of grapes, $\frac{1}{2}$ cup yams/sweet potatoes). Frequency categories vary by food item and range from 'never or less than once per month' to 'five plus per day'. The reproducibility and validity of the YAQ were previously evaluated in youth aged 9-18 years by comparing intake 
determined from the average of three $24 \mathrm{~h}$ recalls and intake determined from two YAQ administered over 1 year $^{(8,9)}$. One-year reproducibility coefficients for energyadjusted nutrient intake had a mean of $0 \cdot 41^{(9)}$. The average correlation coefficient between energy-adjusted nutrient intake determined from the recalls and the average of the two YAQ was $0 \cdot 45^{(8)}$.

\section{Nutrient intake analysis}

Intakes of energy and various nutrients were selected a priori to examine the comparability of estimates determined from the adult FFQ and estimates determined from the YAQ. Nutrients were selected for consideration with an emphasis on outcomes relevant to chronic disease prevention (e.g. Ca, fibre) and reproductive health (e.g. folate, $\mathrm{Fe})^{(16-18)}$. Daily intake estimates for the adult FFQ and the YAQ were determined in 2009 by the Nutrition Questionnaire Service Center at the Harvard School of Public Health using a specially designed database, primarily based on the US Department of Agriculture's Nutrient Database for Standard Reference (release 19) ${ }^{(19)}$.

\section{Definitions for daily food servings}

Foods were grouped into categories for statistical analysis: fruit, whole fruit, fruit juice, vegetables (excluding French fries and potatoes), dark green/orange vegetables, starchy vegetables, grains, whole grains, milk products, snack foods, soft drinks and sugar-sweetened beverages. A daily serving was defined as the equivalent of one-half cup for fruit and vegetables, one cup for milk products and $16 \mathrm{~g}$ for whole grains. For soft drinks and sugarsweetened beverages, a serving was defined as the equivalent of one glass, bottle or can.

\section{Statistical analyses}

Descriptive statistics were calculated to describe daily intakes of energy, nutrients and food servings. Means determined from the adult FFQ and the YAQ were compared using paired $t$ tests for all dietary outcomes with the exception of alcohol; the Wilcoxon signed-rank test was used to examine alcohol intake as the distribution was highly skewed. Testing was carried out using the square root transformation for all intake estimates that exhibited positive skewness. A 95\% confidence level was used to interpret the statistical significance of probability tests, corresponding to a two-sided $P$ value $<0 \cdot 05$. In order to investigate the consistency of intake rankings determined from the adult FFQ and the YAQ, intake estimates for energy, each nutrient and each food category were first classified into quartiles based on the distribution of intake in the sample. The proportion of participants classified in the same quartile and within one quartile based on their responses to the two FFQ was calculated for each dietary intake measure of interest with the exception of alcohol, which was examined by tertiles. Pearson correlations were also calculated for unadjusted intake estimates and intake estimates that were energy-adjusted using the nutrient density method ${ }^{(20)}$. All analyses were conducted using the SAS statistical software package version $9 \cdot 1$ (2002-2003; SAS Institute, Cary, NC, USA).

\section{Results}

\section{Comparability of absolute intake estimates}

Means, medians and interquartile ranges for dietary intake were determined from the adult FFQ and YAQ (Table 1). In general, mean intake estimates determined from the adult FFQ were significantly higher than estimates determined from the YAQ. Only mean estimates for energy from protein, energy from carbohydrate, $\mathrm{Na}$ and total grain servings were found to be comparable.

\section{Comparability of rank classifications}

The percentage of the sample classified into the same quartile rank category based on their responses to the adult FFQ and the YAQ ranged from $31 \%$ for energy from total fat to $63 \%$ for sugar-sweetened drinks (Table 2). Cross-classification to within one quartile was also examined and frank misclassification defined as classification into opposing quartiles (e.g. lowest quartile based on the adult FFQ and highest quartile based on the YAQ). The percentage of the sample that was frankly misclassified ranged from only $6 \%$ for sugar-sweetened drinks to $24 \%$ for grains and energy from carbohydrate. Similar findings were observed when the sample was stratified by gender and age.

As approximately one-third of the overall sample reported consuming alcoholic beverages never or less than once per month, tertiles were used to examine rank classifications for alcohol. The percentage of the overall sample classified into the same tertile rank for alcohol was $80 \%$ and the percentage frankly misclassified was less than $2 \%$ (data not shown). Across all gender and age subgroups, the percentage of the sample classified into the same tertile rank for alcohol ranged from $73 \%$ to $81 \%$ and the percentage frankly misclassified ranged from $1 \%$ to $2 \%$.

\section{Correlations between absolute and energy- adjusted intake estimates}

Pearson correlations between dietary intake estimates determined from the adult FFQ and YAQ were examined for the overall sample, by gender and by age group (Table 3). With few exceptions, correlation coefficients were moderate $(r=0 \cdot 4-0 \cdot 6)$ in the overall sample. Lower correlations were observed for total vitamin C $(r=0 \cdot 28)$, total vitamin $\mathrm{E}(r=0 \cdot 27)$ and total grain servings $(r=0 \cdot 31)$. Similar results were observed when the sample was stratified by gender and age (Table 3), and following adjustment of intake estimates for total energy consumption (data not shown). 
Table 1 Daily intakes of energy, nutrients and food servings estimated by the youth/adolescent and adult FFQ among young adults ( $n$ 194), Minneapolis/St. Paul, MN, USA, 2008-2009

\begin{tabular}{|c|c|c|c|c|c|c|c|c|}
\hline & \multicolumn{4}{|c|}{ Adult form } & \multicolumn{4}{|c|}{ Adolescent form } \\
\hline & Mean & SD & Median & Interquartile ranget & Mean & SD & Median & Interquartile range \\
\hline \multicolumn{9}{|c|}{ ENERGY AND NUTRIENTS } \\
\hline Energy (kJ) & 9353 & 4061 & 8495 & $6259-11187$ & $7787^{\star}$ & 11187 & 7624 & 5715-9282 \\
\hline Energy (kcal) & 2234 & 970 & 2029 & 1495-2672 & $1860^{*}$ & 2672 & 1821 & $1365-2217$ \\
\hline Protein $(\mathrm{g})$ & 93 & 48 & 80 & $57-117$ & $74^{\star}$ & 29 & 70 & 56-89 \\
\hline$\%$ of energy & $16 \cdot 5$ & $3 \cdot 7$ & $16 \cdot 3$ & $13 \cdot 9-18 \cdot 5$ & $16 \cdot 1$ & $3 \cdot 0$ & $15 \cdot 8$ & $14 \cdot 1-17 \cdot 5$ \\
\hline Total fat $(\mathrm{g})$ & 73 & 36 & 67 & $48-91$ & $65^{\star}$ & 28 & 62 & $44-80$ \\
\hline$\%$ of energy & $29 \cdot 5$ & $6 \cdot 5$ & $30 \cdot 0$ & $25 \cdot 6-33 \cdot 0$ & $31 \cdot 4^{*}$ & $5 \cdot 2$ & $32 \cdot 0$ & $27 \cdot 8-34 \cdot 6$ \\
\hline Saturated fat (g) & 25 & 13 & 22 & $16-32$ & $22^{*}$ & 10 & 21 & $15-28$ \\
\hline$\%$ of energy & $10 \cdot 1$ & $2 \cdot 9$ & $10 \cdot 1$ & $8 \cdot 6-11 \cdot 8$ & $10 \cdot 7^{\star}$ & $2 \cdot 4$ & $10 \cdot 7$ & $9 \cdot 1-12 \cdot 2$ \\
\hline Carbohydrate (g) & 295 & 136 & 267 & $194-367$ & $246^{*}$ & 104 & 234 & 175-299 \\
\hline$\%$ of energy & $53 \cdot 0$ & $9 \cdot 0$ & $52 \cdot 5$ & $47 \cdot 7-57 \cdot 5$ & $52 \cdot 8$ & $6 \cdot 9$ & $52 \cdot 9$ & $48 \cdot 4-57 \cdot 4$ \\
\hline Alcohol (g) & $8 \cdot 0$ & $12 \cdot 6$ & $3 \cdot 6$ & $0 \cdot 0-10 \cdot 4$ & $3 \cdot 1^{*}$ & $3 \cdot 4$ & $2 \cdot 2$ & $0 \cdot 0-5 \cdot 3$ \\
\hline Fibre $(\mathrm{g})$ & 21 & 14 & 19 & $11-26$ & $16^{*}$ & 8 & 15 & $9-20$ \\
\hline Folate $(\mu \mathrm{g})$ & 891 & 662 & 660 & $439-1117$ & $625^{\star}$ & 317 & 587 & $372-812$ \\
\hline \multicolumn{9}{|l|}{ Vitamin $A(\mu \mathrm{g})$} \\
\hline Total & 1200 & 990 & 918 & $531-1610$ & $864^{\star}$ & 539 & 705 & $443-1246$ \\
\hline Dietary sources & 866 & 605 & 732 & $458-1054$ & $665^{\star}$ & 388 & 602 & $385-813$ \\
\hline \multicolumn{9}{|l|}{ Vitamin C (mg) } \\
\hline Total & 198 & 230 & 127 & $71-222$ & $101^{*}$ & 63 & 87 & $53-134$ \\
\hline Dietary sources & 125 & 112 & 96 & $52-153$ & $92^{*}$ & 60 & 74 & $47-122$ \\
\hline \multicolumn{9}{|l|}{ Vitamin $D(\mu \mathrm{g})$} \\
\hline Total & 10 & 8 & 7 & $4-13$ & $5^{\star}$ & 4 & 4 & $2-7$ \\
\hline Dietary sources & 7 & 6 & 5 & $3-9$ & $4^{\star}$ & 3 & 3 & $2-4$ \\
\hline \multicolumn{9}{|l|}{ Vitamin $\mathrm{E}(\mathrm{mg})$} \\
\hline Total & 18 & 45 & 6 & $3-14$ & $7^{*}$ & 4 & 6 & $5-9$ \\
\hline Dietary sources & 6 & 4 & 5 & $3-7$ & $6^{*}$ & 3 & 6 & $4-8$ \\
\hline \multicolumn{9}{|l|}{$\mathrm{Ca}(\mathrm{mg})$} \\
\hline Total & 1249 & 782 & 1085 & $681-1694$ & $868^{\star}$ & 493 & 824 & $516-1123$ \\
\hline Dietary sources & 1103 & 685 & 965 & $639-1346$ & $849^{*}$ & 488 & 799 & $496-1103$ \\
\hline \multicolumn{9}{|l|}{$\mathrm{Fe}(\mathrm{mg})$} \\
\hline Total & 20 & 14 & 16 & $11-26$ & $16^{\star}$ & 8 & 15 & $9-21$ \\
\hline Dietary sources & 16 & 10 & 14 & $9-19$ & $13^{*}$ & 6 & 13 & $9-17$ \\
\hline \multicolumn{9}{|l|}{$\mathrm{Zn}(\mathrm{mg})$} \\
\hline Total & 17 & 11 & 14 & $9-22$ & $12^{*}$ & 6 & 11 & $8-16$ \\
\hline Dietary sources & 13 & 7 & 12 & $8-16$ & $10^{*}$ & 5 & 10 & $7-13$ \\
\hline \multicolumn{9}{|l|}{$\mathrm{Mg}(\mathrm{mg})$} \\
\hline Total & 359 & 193 & 322 & $236-479$ & $259^{*}$ & 111 & 250 & $185-322$ \\
\hline Dietary sources & 348 & 183 & 313 & $230-469$ & $254^{*}$ & 109 & 248 & $182-321$ \\
\hline $\mathrm{Na}(\mathrm{mg})$ & 2412 & 1267 & 2111 & $1604-3047$ & 2326 & 1040 & 2233 & 1618-2919 \\
\hline \multicolumn{9}{|c|}{ FOOD GROUPS (servings) } \\
\hline \multicolumn{9}{|l|}{ Fruit } \\
\hline Total & $2 \cdot 1$ & $1 \cdot 9$ & $1 \cdot 7$ & $0 \cdot 8-2 \cdot 9$ & $1 \cdot 5^{\star}$ & $1 \cdot 1$ & $1 \cdot 3$ & $0 \cdot 6-2 \cdot 1$ \\
\hline Whole fruit & $1 \cdot 2$ & $1 \cdot 2$ & 0.9 & $0 \cdot 4-1 \cdot 5$ & $0 \cdot 9^{*}$ & $0 \cdot 7$ & $0 \cdot 8$ & $0 \cdot 4-1 \cdot 4$ \\
\hline Fruit juice & 0.8 & $1 \cdot 0$ & 0.5 & $0 \cdot 2-1 \cdot 1$ & $0 \cdot 6^{*}$ & $0 \cdot 7$ & 0.2 & $0 \cdot 1-1 \cdot 0$ \\
\hline \multicolumn{9}{|l|}{ Vegetables } \\
\hline Total & $2 \cdot 5$ & $2 \cdot 6$ & $1 \cdot 8$ & $0.9-3 \cdot 4$ & $1 \cdot 7^{\star}$ & $1 \cdot 2$ & $1 \cdot 4$ & $0 \cdot 8-2 \cdot 3$ \\
\hline Dark green/orange & $0 \cdot 7$ & 0.9 & 0.5 & $0.2-0.9$ & $0 \cdot 4^{*}$ & $0 \cdot 4$ & $0 \cdot 3$ & $0 \cdot 2-0 \cdot 6$ \\
\hline Starchy & 0.5 & 0.5 & 0.4 & $0 \cdot 2-0 \cdot 7$ & $0 \cdot 3^{*}$ & $0 \cdot 3$ & $0 \cdot 2$ & $0.2-0.5$ \\
\hline Milk products & $2 \cdot 0$ & $1 \cdot 7$ & $1 \cdot 5$ & $0 \cdot 8-2 \cdot 6$ & $2 \cdot 2^{*}$ & 1.5 & 1.9 & $1 \cdot 2-3 \cdot 0$ \\
\hline \multicolumn{9}{|l|}{ Grains } \\
\hline Total & $5 \cdot 6$ & $4 \cdot 3$ & $4 \cdot 7$ & $2 \cdot 9-6 \cdot 7$ & $5 \cdot 4$ & $2 \cdot 4$ & $5 \cdot 0$ & $3 \cdot 6-6 \cdot 9$ \\
\hline Whole grains & $2 \cdot 0$ & $2 \cdot 4$ & $1 \cdot 5$ & $0 \cdot 7-2 \cdot 6$ & $1 \cdot 3^{*}$ & 0.9 & $1 \cdot 1$ & $0 \cdot 6-2 \cdot 0$ \\
\hline Snack foods & 1.9 & $1 \cdot 4$ & $1 \cdot 6$ & $0.9-2 \cdot 4$ & $1 \cdot 6^{*}$ & $1 \cdot 2$ & $1 \cdot 3$ & $0 \cdot 8-2 \cdot 2$ \\
\hline Soft drinks & $1 \cdot 2$ & $1 \cdot 4$ & 0.6 & $0 \cdot 2-1 \cdot 6$ & $0 \cdot 8^{*}$ & $0 \cdot 9$ & 0.6 & $0 \cdot 2-1 \cdot 1$ \\
\hline Sugar-sweetened drinks & 0.9 & $1 \cdot 3$ & 0.3 & $0 \cdot 1-1 \cdot 0$ & $0 \cdot 6^{*}$ & $0 \cdot 8$ & 0.3 & $0.1-0.9$ \\
\hline
\end{tabular}

*Two-sided $P<0.05$ based on a paired $t$ test or Wilcoxon signed-rank test comparing the adult and adolescent form. tThe interquartile range represents the 25 th percentile to the 75 th percentile in the sample population.

\section{Discussion}

In response to the need for population-based research to build a stronger understanding of dietary changes during the transition to young adulthood, the current study describes the comparability of intake estimates that were determined using youth/adolescent and adult forms of the Willett FFQ. The YAQ and adult FFQ were not found to provide comparable estimates of absolute intake. However, for most foods and nutrients examined here, 
Table 2 Concordance of daily intake quartile classifications based on the youth/adolescent and adult FFQ among young adults ( $n$ 194), Minneapolis/St. Paul, MN, USA, 2008-2009

\begin{tabular}{|c|c|c|c|c|c|c|c|c|c|c|}
\hline & \multicolumn{2}{|c|}{ Total $(n$ 194) } & \multicolumn{2}{|c|}{ Male $(n 91)$} & \multicolumn{2}{|c|}{ Female $(n$ 103) } & \multicolumn{2}{|c|}{$20-25$ years $(n 99)$} & \multicolumn{2}{|c|}{$26-31$ years $(n 95)$} \\
\hline & $\begin{array}{c}\% \text { in same } \\
\text { quartilet }\end{array}$ & $\begin{array}{l}\% \text { within one } \\
\text { quartile }\end{array}$ & $\begin{array}{c}\% \text { in same } \\
\text { quartilet }\end{array}$ & $\begin{array}{l}\% \text { within one } \\
\text { quartile }\end{array}$ & $\begin{array}{c}\% \text { in same } \\
\text { quartilet }\end{array}$ & $\begin{array}{l}\% \text { within one } \\
\text { quartile }\end{array}$ & $\begin{array}{c}\% \text { in same } \\
\text { quartilet }\end{array}$ & $\begin{array}{c}\% \text { within one } \\
\text { quartile }\end{array}$ & $\begin{array}{c}\% \text { in same } \\
\text { quartilet }\end{array}$ & $\begin{array}{l}\% \text { within one } \\
\text { quartile }\end{array}$ \\
\hline \multicolumn{11}{|l|}{ ENERGY AND NUTRIENTS } \\
\hline Energy (kJ) & $42 \cdot 8$ & $80 \cdot 4$ & $46 \cdot 1$ & $86 \cdot 8$ & $32 \cdot 0$ & $76 \cdot 7$ & $48 \cdot 5$ & $87 \cdot 9$ & $41 \cdot 0$ & $75 \cdot 8$ \\
\hline Protein (\% of energy) & $41 \cdot 7$ & 81.9 & $39 \cdot 6$ & $85 \cdot 7$ & $40 \cdot 8$ & $79 \cdot 6$ & $38 \cdot 4$ & $79 \cdot 8$ & $44 \cdot 2$ & $82 \cdot 1$ \\
\hline Total fat ( $\%$ of energy) & $31 \cdot 4$ & $77 \cdot 3$ & $30 \cdot 8$ & 76.9 & $34 \cdot 0$ & $77 \cdot 7$ & $23 \cdot 2$ & $71 \cdot 7$ & 38.9 & $82 \cdot 1$ \\
\hline Saturated fat (\% of energy) & $37 \cdot 6$ & $79 \cdot 9$ & $35 \cdot 2$ & $79 \cdot 1$ & 33.0 & $79 \cdot 6$ & $35 \cdot 3$ & $79 \cdot 8$ & $35 \cdot 8$ & $80 \cdot 0$ \\
\hline Carbohydrate ( $\%$ of energy) & $39 \cdot 7$ & $76 \cdot 3$ & $40 \cdot 7$ & 76.9 & $39 \cdot 8$ & $79 \cdot 6$ & $36 \cdot 4$ & $77 \cdot 8$ & $37 \cdot 9$ & 78.9 \\
\hline Fibre $(\mathrm{g})$ & $56 \cdot 8$ & $92 \cdot 8$ & $49 \cdot 4$ & $96 \cdot 7$ & $51 \cdot 5$ & $86 \cdot 4$ & $55 \cdot 6$ & $89 \cdot 9$ & $53 \cdot 7$ & $91 \cdot 6$ \\
\hline Folate $(\mu \mathrm{g})$ & $41 \cdot 2$ & $85 \cdot 6$ & $42 \cdot 9$ & $86 \cdot 8$ & $44 \cdot 7$ & $86 \cdot 4$ & $39 \cdot 4$ & $84 \cdot 8$ & $42 \cdot 1$ & $87 \cdot 4$ \\
\hline \multicolumn{11}{|l|}{ Vitamin $A(\mu g)$} \\
\hline Total & $55 \cdot 6$ & $91 \cdot 9$ & $54 \cdot 4$ & $87 \cdot 8$ & $44 \cdot 7$ & $85 \cdot 4$ & $45 \cdot 9$ & $85 \cdot 7$ & $47 \cdot 9$ & $86 \cdot 2$ \\
\hline Dietary sources & $46 \cdot 9$ & $83 \cdot 0$ & $47 \cdot 2$ & $89 \cdot 0$ & $49 \cdot 5$ & $78 \cdot 6$ & $46 \cdot 5$ & $80 \cdot 8$ & $46 \cdot 3$ & $85 \cdot 3$ \\
\hline \multicolumn{11}{|l|}{ Vitamin C (mg) } \\
\hline Total & $46 \cdot 9$ & $86 \cdot 6$ & $46 \cdot 1$ & $87 \cdot 9$ & $42 \cdot 7$ & $83 \cdot 5$ & $42 \cdot 4$ & $86 \cdot 9$ & $46 \cdot 3$ & $85 \cdot 3$ \\
\hline Dietary sources & $41 \cdot 2$ & $85 \cdot 6$ & $49 \cdot 4$ & $87 \cdot 9$ & $37 \cdot 9$ & $84 \cdot 5$ & $44 \cdot 4$ & $84 \cdot 8$ & 38.9 & $85 \cdot 3$ \\
\hline \multicolumn{11}{|l|}{ Vitamin D $(\mu \mathrm{g})$} \\
\hline Total & $44 \cdot 3$ & $87 \cdot 1$ & $42 \cdot 9$ & $87 \cdot 9$ & $48 \cdot 5$ & $89 \cdot 3$ & $47 \cdot 5$ & $89 \cdot 9$ & $40 \cdot 0$ & $85 \cdot 3$ \\
\hline Dietary sources & $40 \cdot 2$ & $86 \cdot 1$ & $40 \cdot 7$ & $90 \cdot 1$ & 36.9 & $81 \cdot 5$ & $43 \cdot 4$ & 86.9 & $36 \cdot 8$ & $87 \cdot 4$ \\
\hline \multicolumn{11}{|l|}{ Vitamin E (mg) } \\
\hline Total & $51 \cdot 0$ & $85 \cdot 6$ & $47 \cdot 2$ & $85 \cdot 7$ & $49 \cdot 5$ & $81 \cdot 5$ & 51.5 & $89 \cdot 9$ & $49 \cdot 5$ & $83 \cdot 1$ \\
\hline Dietary sources & $40 \cdot 7$ & $80 \cdot 9$ & $45 \cdot 0$ & $90 \cdot 1$ & $34 \cdot 0$ & $78 \cdot 6$ & $41 \cdot 4$ & $77 \cdot 8$ & $41 \cdot 0$ & $85 \cdot 3$ \\
\hline \multicolumn{11}{|l|}{$\mathrm{Ca}(\mathrm{mg})$} \\
\hline Total & $49 \cdot 0$ & $88 \cdot 1$ & 54.9 & $89 \cdot 0$ & $44 \cdot 7$ & 83.5 & $50 \cdot 5$ & $89 \cdot 9$ & $49 \cdot 5$ & $84 \cdot 2$ \\
\hline Dietary sources & $53 \cdot 6$ & $88 \cdot 1$ & $57 \cdot 1$ & $91 \cdot 2$ & $47 \cdot 6$ & 83.5 & $55 \cdot 6$ & $87 \cdot 9$ & $48 \cdot 4$ & $87 \cdot \overline{4}$ \\
\hline \multicolumn{11}{|l|}{$\mathrm{Fe}(\mathrm{mg})$} \\
\hline Total & $46 \cdot 4$ & $84 \cdot 0$ & $46 \cdot 1$ & $86 \cdot 8$ & $45 \cdot 6$ & $84 \cdot 5$ & $50 \cdot 5$ & 85.9 & $44 \cdot 2$ & $82 \cdot 1$ \\
\hline Dietary sources & $47 \cdot 9$ & $85 \cdot 6$ & $52 \cdot 7$ & $86 \cdot 8$ & $44 \cdot 7$ & 83.5 & $51 \cdot 5$ & $84 \cdot 8$ & $47 \cdot 4$ & $88 \cdot 4$ \\
\hline \multicolumn{11}{|l|}{$\mathrm{Zn}(\mathrm{mg})$} \\
\hline Total & $48 \cdot 6$ & 81.5 & $44 \cdot 0$ & 83.5 & $48 \cdot 5$ & 84.5 & $54 \cdot 5$ & 85.9 & $43 \cdot 2$ & $86 \cdot 3$ \\
\hline Dietary sources & $46 \cdot 4$ & $87 \cdot 6$ & $52 \cdot 7$ & $85 \cdot 7$ & $44 \cdot 7$ & $85 \cdot 4$ & $48 \cdot 5$ & $90 \cdot 9$ & $48 \cdot 4$ & $84 \cdot 2$ \\
\hline \multicolumn{11}{|l|}{$\mathrm{Mg}(\mathrm{mg})$} \\
\hline Total & $47 \cdot 9$ & $87 \cdot 1$ & $53 \cdot 8$ & $93 \cdot 4$ & $43 \cdot 7$ & $80 \cdot 6$ & $56 \cdot 6$ & 85.9 & $42 \cdot 1$ & 89.5 \\
\hline Dietary sources & $48 \cdot 4$ & $86 \cdot 6$ & $50 \cdot 5$ & $92 \cdot 3$ & $39 \cdot 8$ & $82 \cdot 5$ & $50 \cdot 5$ & $87 \cdot 9$ & 38.9 & $87 \cdot 4$ \\
\hline $\mathrm{Na}(\mathrm{mg})$ & $45 \cdot 9$ & $82 \cdot 0$ & $45 \cdot 0$ & $87 \cdot 9$ & $41 \cdot 7$ & $79 \cdot 6$ & $47 \cdot 5$ & $88 \cdot 9$ & $44 \cdot 2$ & $78 \cdot 9$ \\
\hline \multicolumn{11}{|l|}{ FOOD GROUPS (servings) } \\
\hline \multicolumn{11}{|l|}{ Fruit } \\
\hline Total & $47 \cdot 9$ & $87 \cdot 6$ & $48 \cdot 3$ & $84 \cdot 6$ & $46 \cdot 6$ & $92 \cdot 2$ & $58 \cdot 6$ & $87 \cdot 9$ & $44 \cdot 2$ & $86 \cdot 3$ \\
\hline Whole fruit & $50 \cdot 5$ & $88 \cdot 7$ & $46 \cdot 1$ & $81 \cdot 3$ & $53 \cdot 4$ & $93 \cdot 2$ & $49 \cdot 5$ & $89 \cdot 9$ & $50 \cdot 5$ & $88 \cdot 4$ \\
\hline Fruit juice & $43 \cdot 5$ & $87 \cdot 0$ & $44 \cdot 4$ & $91 \cdot 1$ & $44 \cdot 7$ & $85 \cdot \overline{4}$ & $45 \cdot 9$ & $89 \cdot 8$ & $47 \cdot 4$ & $84 \cdot 2$ \\
\hline \multicolumn{11}{|l|}{ Vegetables } \\
\hline Total & $54 \cdot 6$ & $89 \cdot 2$ & $64 \cdot 8$ & $91 \cdot 2$ & $50 \cdot 5$ & $90 \cdot 3$ & $49 \cdot 5$ & $83 \cdot 8$ & $57 \cdot 9$ & $93 \cdot 7$ \\
\hline Dark green/orange & $51 \cdot 0$ & $88 \cdot 1$ & 54.9 & $84 \cdot \overline{6}$ & 48.5 & $87 \cdot 4$ & $52 \cdot 5$ & $88 \cdot 9$ & $49 \cdot 5$ & $90 \cdot 5$ \\
\hline Starchy & $46 \cdot 9$ & $86 \cdot 6$ & $44 \cdot 0$ & $90 \cdot 2$ & $46 \cdot 6$ & 84.5 & $54 \cdot 5$ & $90 \cdot 9$ & $42 \cdot 1$ & $84 \cdot 2$ \\
\hline Milk products & $50 \cdot 5$ & $87 \cdot 6$ & $49 \cdot 4$ & $90 \cdot 1$ & $51 \cdot 5$ & $88 \cdot 4$ & $53 \cdot 5$ & $88 \cdot 9$ & $46 \cdot 3$ & $85 \cdot 3$ \\
\hline \multicolumn{11}{|l|}{ Grains } \\
\hline Total & $39 \cdot 1$ & $76 \cdot 3$ & $46 \cdot 1$ & $79 \cdot 1$ & $31 \cdot 1$ & $69 \cdot 9$ & $44 \cdot 4$ & $76 \cdot 8$ & $37 \cdot 9$ & $73 \cdot 7$ \\
\hline Whole grains & $50 \cdot 0$ & $90 \cdot 7$ & $60 \cdot 4$ & $93 \cdot 4$ & $40 \cdot 8$ & $88 \cdot 3$ & $45 \cdot 5$ & $85 \cdot 8$ & $50 \cdot 0$ & $94 \cdot 7$ \\
\hline Snack foods & $42 \cdot 8$ & $84 \cdot 0$ & $47 \cdot 2$ & $86 \cdot 8$ & $40 \cdot 8$ & $80 \cdot 6$ & $35 \cdot 3$ & $77 \cdot 8$ & $43 \cdot 2$ & $90 \cdot 5$ \\
\hline Soft drinks & $53 \cdot 6$ & $93 \cdot 3$ & $52 \cdot 7$ & $90 \cdot 1$ & $53 \cdot 4$ & $92 \cdot 2$ & $55 \cdot 6$ & 93.9 & $56 \cdot 8$ & $88 \cdot 4$ \\
\hline Sugar-sweetened drinks & $62 \cdot 9$ & $93 \cdot 8$ & $50 \cdot 5$ & $96 \cdot 7$ & $56 \cdot 3$ & $91 \cdot 3$ & $54 \cdot 5$ & 93.9 & $65 \cdot 3$ & $93 \cdot 7$ \\
\hline
\end{tabular}


Table 3 Pearson correlations between daily intaket estimates as assessed with the youth/adolescent and adult FFQ among young adults ( $n$ 194), Minneapolis/St. Paul, MN, USA, 2008-2009

\begin{tabular}{|c|c|c|c|c|c|}
\hline & $\begin{array}{l}\text { Total } \\
(n \text { 194 })\end{array}$ & $\begin{array}{l}\text { Male } \\
(n 91)\end{array}$ & $\begin{array}{l}\text { Female } \\
(n \text { 103) }\end{array}$ & $\begin{array}{c}20-25 \text { years } \\
(n 99)\end{array}$ & $\begin{array}{c}26-31 \text { years } \\
(n \text { 95) }\end{array}$ \\
\hline \multicolumn{6}{|c|}{ ENERGY AND NUTRIENTS } \\
\hline Energy (kJ) & 0.51 & $0 \cdot 61$ & 0.35 & 0.59 & 0.44 \\
\hline Protein (g) & 0.50 & 0.54 & 0.43 & 0.54 & $0 \cdot 47$ \\
\hline Total fat $(\mathrm{g})$ & 0.51 & 0.56 & 0.43 & 0.59 & 0.44 \\
\hline Saturated fat (g) & 0.53 & 0.57 & 0.47 & 0.59 & 0.48 \\
\hline Carbohydrate (g) & 0.48 & $0 \cdot 62$ & $0 \cdot 30$ & 0.52 & 0.45 \\
\hline Alcohol (g) & 0.63 & $0 \cdot 60$ & $0 \cdot 74$ & $0 \cdot 62$ & 0.64 \\
\hline Fibre $(\mathrm{g})$ & $0 \cdot 61$ & 0.69 & 0.50 & $0 \cdot 61$ & $0 \cdot 61$ \\
\hline Folate $(\mu \mathrm{g})$ & 0.53 & 0.55 & 0.51 & 0.48 & 0.57 \\
\hline \multicolumn{6}{|l|}{ Vitamin A $(\mu \mathrm{g})$} \\
\hline Total & 0.50 & 0.57 & 0.44 & 0.47 & 0.52 \\
\hline Dietary sources & 0.45 & 0.56 & $0 \cdot 38$ & 0.54 & 0.38 \\
\hline \multicolumn{6}{|l|}{ Vitamin C (mg) } \\
\hline Total & $0 \cdot 28$ & 0.33 & $0 \cdot 24$ & $0 \cdot 30$ & 0.27 \\
\hline Dietary sources & 0.42 & 0.50 & $0 \cdot 39$ & 0.45 & $0 \cdot 40$ \\
\hline \multicolumn{6}{|l|}{ Vitamin D $(\mu \mathrm{g})$} \\
\hline Total & 0.51 & 0.46 & 0.57 & 0.57 & 0.47 \\
\hline Dietary sources & $0 \cdot 46$ & 0.47 & 0.44 & $0 \cdot 48$ & 0.45 \\
\hline \multicolumn{6}{|l|}{ Vitamin E (mg) } \\
\hline Total & $0 \cdot 27$ & $0 \cdot 35$ & $0 \cdot 17$ & 0.35 & $0 \cdot 20$ \\
\hline Dietary sources & $0 \cdot 48$ & $0 \cdot 69$ & $0 \cdot 29$ & 0.45 & 0.52 \\
\hline \multicolumn{6}{|l|}{$\mathrm{Ca}(\mathrm{mg})$} \\
\hline Total & 0.59 & 0.63 & 0.53 & 0.63 & 0.56 \\
\hline Dietary sources & 0.62 & 0.67 & 0.54 & 0.62 & 0.62 \\
\hline \multicolumn{6}{|l|}{$\mathrm{Fe}(\mathrm{mg})$} \\
\hline Total & 0.52 & $0 \cdot 60$ & 0.47 & 0.48 & 0.58 \\
\hline Dietary sources & 0.48 & 0.63 & $0 \cdot 36$ & 0.43 & 0.57 \\
\hline \multicolumn{6}{|l|}{$\mathrm{Zn}(\mathrm{mg})$} \\
\hline Total & 0.47 & 0.41 & 0.55 & 0.45 & 0.49 \\
\hline Dietary sources & 0.59 & 0.62 & 0.56 & 0.58 & $0 \cdot 60$ \\
\hline \multicolumn{6}{|l|}{$M g(m g)$} \\
\hline Total & $0 \cdot 60$ & 0.69 & 0.48 & $0 \cdot 62$ & 0.59 \\
\hline Dietary sources & $0 \cdot 60$ & 0.69 & 0.48 & $0 \cdot 62$ & 0.58 \\
\hline $\mathrm{Na}(\mathrm{mg})$ & 0.53 & 0.58 & $0 \cdot 43$ & 0.57 & $0 \cdot 49$ \\
\hline \multicolumn{6}{|l|}{ FOOD GROUPS (servings) } \\
\hline \multicolumn{6}{|l|}{ Fruit } \\
\hline Total & 0.51 & 0.55 & 0.50 & $0 \cdot 60$ & 0.43 \\
\hline Whole fruit & 0.51 & 0.53 & 0.51 & 0.57 & $0 \cdot 49$ \\
\hline Fruit juice & 0.55 & 0.59 & 0.53 & 0.63 & 0.44 \\
\hline \multicolumn{6}{|l|}{ Vegetables } \\
\hline Total & 0.49 & 0.59 & 0.41 & 0.47 & 0.51 \\
\hline Dark green/orange & 0.50 & 0.57 & 0.46 & 0.58 & 0.46 \\
\hline Starchy & 0.50 & 0.47 & 0.50 & 0.50 & 0.51 \\
\hline Milk products & 0.61 & 0.63 & 0.56 & $0 \cdot 60$ & $0 \cdot 61$ \\
\hline \multicolumn{6}{|l|}{ Grains } \\
\hline Total & $0 \cdot 31$ & $0 \cdot 39$ & $0 \cdot 16$ & $0 \cdot 33$ & $0 \cdot 30$ \\
\hline Whole grains & 0.59 & 0.58 & 0.65 & 0.55 & $0 \cdot 65$ \\
\hline Snack foods & 0.55 & 0.68 & $0 \cdot 38$ & 0.56 & 0.57 \\
\hline Soft drinks & 0.55 & 0.63 & 0.50 & $0 \cdot 69$ & 0.43 \\
\hline Sugar-sweetened drinks & $0 \cdot 62$ & $0 \cdot 67$ & 0.58 & 0.73 & 0.51 \\
\hline
\end{tabular}

tIntake is not adjusted for total energy consumption.

correlations between intake estimates determined from the YAQ and adult FFQ were moderate. Further, the percentage of individuals classified into the same quartile rank category based on their responses to the YAQ and adult FFQ was $50 \%$ or greater for fibre, vitamins $\mathrm{A}$ and $\mathrm{E}$, and servings of fruit (excluding juice), vegetables, dairy, whole grains, soft drinks and sugar-sweetened beverages.

Absolute intake estimates based on responses to the adult FFQ were generally higher than intake estimates based on responses to the YAQ. This discrepancy is likely due to the overall greater number of food items included on the adult FFQ relative to the YAQ (151 items $v .127$ items). Particularly notable differences were found for fruits and vegetables. The adult FFQ includes sixteen fruit items and twenty-six vegetable items while the YAQ includes only eleven fruit items and nineteen vegetable items. Prior research has similarly shown that fruit and vegetable intake estimates are directly related to the number of food items assessed ${ }^{(21,22)}$.

Other key differences between the YAQ and adult FFQ that may have limited the comparability of intake 
estimates relate to the assessment of dietary supplements and reduced-fat forms of food. Mean intake estimates determined from the adult FFQ were greater than intake estimates determined from the YAQ for all vitamins and minerals examined here. While both the YAQ and adult FFQ assess the use of multi-vitamin preparations, the discrepancy may be partially explained by the additional assessment of multiple specific vitamin and mineral preparations on the adult FFQ. In contrast, mean estimates of total fat and saturated fat determined from the adult FFQ were lower than intake estimates determined from the YAQ. This difference may be in part due to the distinction between regular and low-fat forms of several foods on the adult FFQ.

Response reliability is a common limitation of the FFQ approach to measuring dietary intake and should be considered when interpreting the results described here ${ }^{(23)}$. The YAQ and adult FFQ were both designed to assess usual intake during the past year and have been shown to provide estimates of dietary intake with acceptable reliability ${ }^{(9,14)}$. However, reported 1-year reproducibility coefficients for the adult FFQ were mostly moderate $(r=0 \cdot 5-0 \cdot 7)$ and 1 -year reproducibility coefficients for the YAQ had a mean of only $0 \cdot 41$. While all participants in the current study completed the adult FFQ and YAQ within a period of 3 weeks, it is possible that similarly moderate correlations between intake estimates would be observed if the participants had instead completed the same FFQ twice.

In drawing conclusions from the present study, certain strengths and limitations of the design should be considered. The young adult sample had participated in up to three surveys as part of a 10-year longitudinal study designed to examine factors that influence weight status and weight-related behaviours such as dietary intake. Although the sample was diverse in terms of race/ethnicity, age and weight status, participants in the current study were potentially more aware of their food choices than the general population of US young adults. It is also possible that some observed differences may be attributable to the mode of administration. The majority of participants (94\%) completed the online form of the adult FFQ while the YAQ was only administered by mail as a paper form; thus, observed correlations may have been higher if both FFQ were completed by the same mode. Further, it is possible the YAQ does not fully reflect the foods and beverages that may be consumed in a typical adult diet as it does not name several foods which are included on the adult FFQ (e.g. onions, avocados, blueberries, breakfast bars, dairy coffee drinks). Examining differences between the YAQ and adult FFQ in a sample of young adults may have led to an underestimate of their true comparability for longitudinal studies of the transition from adolescence to adulthood.

Study findings indicate that future longitudinal research can use the YAQ and adult FFQ together to investigate patterns of dietary intake during the transition from adolescence to young adulthood. While these tools cannot be used to describe changes over time in the absolute amount of foods or nutrients usually consumed, the YAQ and adult FFQ may be used together to examine relative tracking over time and to investigate influences on dietary intake. When the YAQ is used to assess diet in adolescence and the adult FFQ is used to assess diet in young adulthood, future studies can examine how the dietary intake of individuals tracks relative to other members of a population during the transition to adulthood. Further, predictors of dietary intake in young adulthood can be examined while accounting for intake patterns in adolescence. Few longitudinal studies have investigated what factors influence dietary intake during this critical period of development and the results described here may be used to inform the design of research to fill this gap.

\section{Acknowledgements}

This work was supported by grant number R01HL084064 from the National Heart, Lung, and Blood Institute (Principal Investigator: D.N.-S.). The content is solely the responsibility of the authors and does not necessarily represent the official views of the National Heart, Lung, and Blood Institute or the National Institutes of Health. The authors have no conflicts of interest to report. N.L. conducted the statistical analysis and drafted the manuscript. L.H. helped to conceptualize the analysis plan. D.N.-S. conceptualized the larger Project EAT-III study design and oversaw data collection. All authors contributed to the interpretation of results and manuscript revisions.

\section{References}

1. Gordon-Larsen P, The N \& Adair L (2010) Longitudinal trends in obesity in the United States from adolescence to the third decade of life. Obesity (Silver Spring) 18, 1801-1804.

2. Gordon-Larsen P, Adair L, Nelson M et al. (2004) Five-year obesity incidence in the transition period between adolescence and adulthood: the National Longitudinal Study of Adolescent Health. Am J Clin Nutr 80, 569-575.

3. Nelson MC, Story M, Larson NI et al. (2008) Emerging adulthood and college-aged youth: an overlooked age for weight-related behavior change. Obesity (Silver Spring) 16, 2205-2211.

4. Fowles E, Sterling B \& Walker L (2007) Measuring dietary intake in nursing research. Can J Nurs Res 39, 146-165.

5. Livingstone $\mathrm{M}$, Robson $\mathrm{P} \&$ \& Wallace $\mathrm{J}$ (2004) Issues in dietary intake assessment of children and adolescents. BrJ Nutr 92, Suppl. 2, S213-S222.

6. Food Surveys Research Group, US Department of Agriculture, Agricultural Research Service (2010) Table 9. Away from home: percentages of selected nutrients contributed by foods eaten away from home, by gender and age, in the United States, 2007-2008. What We Eat in America, NHANES, 2007-2008. http://www.ars.usda.gov/Services/ docs.htm?docid=18349 (accessed July 2011).

7. Cade J, Thompson R, Burley V et al. (2002) Development, validation and utilization of food-frequency questionnaires - a review. Public Health Nutr 5, 567-587.

8. Rockett H, Breitenbach M, Frazier A et al. (1997) Validation of a youth/adolescent food frequency questionnaire. Prev Med 26, 808-816. 
9. Rockett H, Wolf A \& Colditz G (1995) Development and reproducibility of a food frequency questionnaire to assess diets of older children and adolescents. J Am Diet Assoc 95, 336-340.

10. Neumark-Sztainer D, Story M, Hannan P et al. (2002) Overweight status and eating patterns among adolescents: where do youth stand in comparison to the Healthy People 2010 Objectives? Am J Public Health 92, 844-851.

11. Neumark-Sztainer D, Croll J, Story M et al. (2002) Ethnic/ racial differences in weight-related concerns and behaviors among adolescent girls and boys: findings from Project EAT. J Psychosom Res 53, 963-974.

12. Larson N, Neumark-Sztainer D, Harwood E et al. (2011) Do young adults participate in surveys that 'go green'? Response rates to a web and mailed survey of weight-related health behaviors. Int J Child Health Hum Dev (In the Press).

13. Harvard School of Public Health Nutrition Department (2011) HSPH Nutrition Department's File Download Site. https:// regepi.bwh.harvard.edu/health/nutrition.html (accessed July 2011).

14. Rimm E, Giovannucci E, Stampfer M et al. (1992) Reproducibility and validity of an expanded self-administered semiquantitative food frequency questionnaire among male health professionals. Am J Epidemiol 135, 1114-1126.

15. Feskanich D, Rimm E, Giovannucci E et al. (1993) Reproducibility and validity of food intake measurements from a semiquantitative food frequency questionnaire. J Am Diet Assoc 93, 790-796.

16. Kumanyika S, Obarzanek E, Stettler N et al. (2008) Populationbased prevention of obesity. The need for comprehensive promotion of healthful eating, physical activity, and energy balance. A scientific statement from American Heart Association Council on Epidemiology and Prevention, Interdisciplinary Committee for Prevention. Circulation 118, 428-464.

17. Houghton L \& O'Connor D (2008) Pregnancy and lactation. In: Optimizing Women's Health through Nutrition, 1st ed., pp. 65-92 [L Thompson and W Ward, editors]. Boca Raton, FL: CRC Press.

18. Dietary Guidelines Advisory Committee, US Department of Health and Human Services, US Department of Agriculture (2011) Report of the Dietary Guidelines Advisory Committee on the Dietary Guidelines for Americans. http://www.cnpp.usda.gov/DGAs2010-DGACReport.htm (accessed July 2011)

19. US Department of Agriculture, Agricultural Research Service (2010) USDA National Nutrient Database for Standard Reference, Release 19. Nutrient Data Laboratory Home Page. http://www.ars.usda.gov/Services/docs.htm? docid $=15973$ (accessed July 2011).

20. Willett WC, Howe GR \& Kushi LH (1997) Adjustment for total energy intake in epidemiologic studies. Am J Clin Nutr 65, 4 Suppl., 1220S-1228S; 12295-12315.

21. Smith-Warner S, Elmer P, Fosdick L et al. (1997) Reliability and comparability of three dietary assessment methods for estimating fruit and vegetable intakes. Epidemiology 8 , 196-201.

22. Thompson F, Kipnis V, Subar A et al. (2000) Evaluation of 2 brief instruments and a food-frequency questionnaire to estimate daily number of servings of fruit and vegetables. Am J Clin Nutr 71, 1503-1510.

23. Willett W (1998) Nutritional Epidemiology, 2nd ed., New York: Oxford University Press. 